\title{
Program assessment of the introduction of multiload-375 IUD in the family welfare program of the government of India*
}

\author{
Basu $\mathrm{S}^{1 *}$, Green $\mathrm{M}^{2}$, Stanback $\mathrm{J}^{3}$ and George $\mathrm{B}^{4}$ \\ ${ }^{1}$ Previously with FHI 360, India \\ ${ }^{2}$ Previously with FHI 360, North Carolina, USA \\ ${ }^{3}$ FHI 360, North Carolina, USA \\ ${ }^{4}$ FHI360, New Delhi, India \\ *Paper presented in the oral session of 2011 International Conference on Family Planning, Dakar, Senegal
}

\begin{abstract}
Background: The Government of India is attempting to revive the IUD which is underutilized in India by introducing the Multiload-375 (ML-375) IUD. This study aimed to identify operational issues associated with the introduction of the ML 375 in public health facilities.
\end{abstract}

Study Design: The study was conducted across six states in India in a District Hospital and a Primary Health Centre. Intervention activities included development of communication materials, a record-keeping system, and trainings on ML 375. In-depth interviews were conducted later with service providers (n=66).

Results: Minimal training was needed to introduce the ML 375 because providers had previous experience with the CuT380A. About $62 \%$ providers preferred the insertion technique of the ML 375 compared to the CuT380A. Myths and misconceptions among clients and providers and weak IUD counseling were barriers.

Conclusions: Improvements in infrastructure, supply chain, patient follow-up, and demand-generation are needed to increase uptake of ML 375 in India.

\section{Introduction}

India and family planning

In 1951, India was the first country in the developing world to initiate a state-sponsored family welfare program with the goal of lowering fertility and slowing the population growth rate. The national government set method-specific targets and promoted sterilization vigorously, resulting in a narrow range of family planning options and limited choice for clients. Currently, however, the government aims to offer high-quality, client-centered, demand-driven, and decentralized contraceptive services among eligible clients, with an emphasis on adequate spacing of births [1].

Overall, India's family welfare program has been successful in lowering fertility rates across the country. Countrywide, total fertility has decreased from 6.6 lifetime births per woman in the early 1970 s to 2.7 lifetime births per woman in 2005-2006 [2]. According to the recent National Family Health Survey data, $37.3 \%$ of currently married women are sterilized [2]. Condoms are the second most frequently used modern family planning method (5.2\%), followed by oral contraceptive pills (3.1\%), and the intrauterine contraceptive device (IUD) (1.7\%). This contraceptive method mix, skewed toward female sterilization, can be a concern when women want only to delay their next pregnancy rather than cease childbearing altogether. Additionally, ensuring that women and their partners have access to a wide range of family planning methods increases their likelihood of choosing a method and results in higher contraceptive use $[3,4]$; increased use reduces unplanned pregnancies, unsafe abortions, and maternal morbidity and mortality $[4,5]$.

\section{The IUD}

The IUD is the most widely used reversible method of contraception worldwide [5,6]. IUDs come in two types, either copper-bearing or hormonal levonorgestrel-releasing, and they provide a safe and highly effective method of contraception of long duration and high efficacy. The copper-bearing IUDs-the Copper $\mathrm{T}$ 380A (CuT 380A) and Multiload 375 (ML 375)-are approvedfor ten years and five years of use respectively; the hormonal levonorgestrel-releasing IUD can be used for up to five years. IUDs, unlike sterilization, are quickly reversible, and hence can meet the needs of both women who want to postpone their next pregnancy and those who want to end childbearing altogether. Furthermore, because IUDs can be used for several years, they are a very cost-effective method of contraception [7]. In many countries, the low use of IUDs can be attributed to outdated safety concerns and misconceptions among providers and clients, as well as

${ }^{\star}$ Correspondence to: Sharmistha Basu, PhD, Programme Officer II, Population Council, New Delhi, India, Tel: +91 9868492880; E-mail: basu_jnu@yahoo.com

Key words: contraception, family planning, india, intervention, service delivery, IUD, Multiload-375, India, operations research

Received: October 08, 2018; Accepted: October 19, 2018; Published: October 23,2018 
to infrastructure and resource challenges such as the need for supplies, equipment, and trained and motivated providers [7].

\section{The IUD in India}

With the approval of the Indian Council of Medical Research, the Lippes Loop was the first IUD to be introduced into the Family Welfare Program (FWP) of India in 1965. This was followed by the introduction of the Copper T 200B in 1975, which was then replaced by the CuT $380 \mathrm{~A}$ in 2002 [1]. Despite its high degree of safety and effectiveness, the IUD remains an underused method of contraception in India. Use among married women of reproductive age remains below $2 \%$ in the country [2]. This low use is attributed to various factors, which include the lack of trained providers, poor quality of IUD services, provider bias against IUDs, and misconceptions and lack of awareness about the method among both clients and health care providers [1,8-10]. Moreover, use of the public sector for IUDs services is limited, and in the past decade there has been a major shift toward seeking IUD services in the private sector.

Beginning in 2006, the Ministry of Health and Family Welfare (MOHFW) started taking steps to revive and reposition the IUD in the country, particularly in states with low contraceptive prevalence rates [1]. Key strategies for the repositioning include enlisting policy support; ensuring the availability of skilled service providers; strengthening infrastructure and logistics for providing quality care; creating awareness and demand in the community; promoting public-private partnerships; and developing a strong monitoring and evaluation system. The MOHFW also began exploring new opportunities for enhancing IUD use and decided to include another type of IUD, the ML 375, in the FWP. The ML 375 IUD is currently available in the private sector and is popular and well-regarded among providers and clients. The ML 375 comes packaged pre-loaded on its inserter. With this advantage, and to capitalize on the ML 375's popularity in the private sector, the MOHFW sought to include the ML 375 IUD in the public sector's contraceptive method mix.

Our study aimed to identify operational issues like ML 375 service delivery aspects such as counseling, service provision, following up of clients, training, intervention communication materials, barriers, and necessary facility- and community-based services. associated with [1] the introduction of the ML 375 in Government of India FWP-supported health facilities in pilot sites and then [2] the initiative's national rollout. The specific objectives of the study were to:

1. Identify operational issues associated with the introduction of the ML 375 into facilities already offering the CuT 380 A under the FWP.

2. Identify barriers to access, uptake, and use of the ML 375 and suggest measures to facilitate uptake.

3. Identify appropriate community- and facility-based services that will be required for the uptake of the ML 375

This assessment is helpful in understanding operational issues in the introduction of a new type of IUD in the FWP along with an existing IUD. Insight gained can help expand the contraceptive method mix and increase the options available to women who want to delay childbearing and adequately space births.

\section{Materials and methods}

The current assessment was based in six states across India in one District Hospital (DH) and one Primary Health Center (PHC) each, $(n=12)$ within the existing government health systems. The six states were Assam (Kamrup district), Gujarat (Gandhinagar district), Jharkhand (Hazaribagh district), Karnataka (Mysore district), Uttar Pradesh (Varanasi district), and West Bengal (Nadia district). Study sites were purposively selected by the Family Planning Division (FPD) of the MOHFW to be geographically representative of the country. Since the evaluation of the introduction was for descriptive purposes only, formal sample size calculations were not undertaken, and facility selection was purposive. Facilities were included in the study if:

\section{Staff had received training in the CuT 380A IUD in the past}

\section{The trained personnel were still working at the facilities}

\section{The CuT 380A IUD was being inserted routinely on-site}

If there was more than one $\mathrm{DH}$ in a district and they all met these criteria, the facility with the highest number of registered IUD/family planning related cases in the past six months was chosen. The same procedure was followed for multiple PHCs meeting all the selection criteria in a district. The Chief District Medical Officer (CDMO) in each district was consulted before the facility selections were made final.

The study included three phases: pre-intervention, intervention, and post-intervention. In the pre-intervention phase, health facility assessments $(n=12)$ and in-depth qualitative interviews $(n=66)$ with facility staff were undertaken from September 2009 to March 2010. The health facility assessment was conducted at each of the chosen 12 health facilities to understand the infrastructure and resources available for the introduction and to document existing family planning services with the help of structured tools which included observations and interviews with service providers at the facility. The data collection, transcription, translation and data entry were undertaken by a research agency, with FHI360 conducting all analyses. The results of the analyses are descriptive and conceptually representative of the types of providers interviewed in the 12 facilities and can be transferred to similar contexts.

The seven-month intervention phase (March-October 2010) activities included training of providers on ML 375 insertion and provision of ML 375 services to clients at the study sites. In addition, communication materials on client education were developed as were record-keeping systems to track intervention activities. At pilot sites, providers began offering the ML 375 in June 2010, and sites were monitored through the end of September 2010. The development of the ML 375 IUD introduction materials was based on MOHFW IUD guidelines. All intervention materials were translated into Hindi and four other regional languages and pretested before final printing and distribution to facilities and staff. The intervention training materials included two manuals. The Trainer's Manual on IUD was developed for the intervention agency lead trainers to train trainers from each study district. The manual introduces the ML 375 study and reviewed general family planning communication and counseling skills, record keeping, and data management. Technical information covers both the ML 375 and CuT 380A IUDs equally. The other training manual was the Handbook for Service Providers on IUD which was developed for the service providers eligible to insert IUDs and trained to the insert the ML 375 during the intervention. The handbook is a concise technical manual on overall IUD provision and provides equally detailed information on both the ML 375 and CuT 380A IUDs. The Intervention communication materials consisted of: Flipbook for Counselors on IUD: This flipbook was developed for use by the community motivators (community-based health workers) and counselors associated with the 12 study facilities and surrounding communities. Motivators and counselors (not qualified to insert IUDs), during the intervention phase introduced IUDs and the ML 375 to women in their communities and 
to provide referrals for interested women. The leaflet was designed to be distributed in communities surrounding the 12 facilities. The leaflet briefly introduces both the ML 375 and CuT 380A IUDs, and also provides general IUD information on how it is used, the benefits and precautions of use, continuation, follow- up, and places where an IUD can be obtained.

The trainings for the ML 375 introduction involved two different groups of service providers: those providers qualified to insert IUDs and community motivators and counselors. Trainings for both types of staff followed a cascade approach, beginning with the private intervention agency identifying and orienting three of their own Lead Trainers who had training and CuT 380A insertion experience. These three individuals trained seven Trainers, approximately one from each study district selected by State government officials, who were OB/Gyns or MOs and had been trained in the past to provide CuT 380A IUDs. The training of the trainers took place over three days and the content covered the Master Trainer's Manual on the IUD and hands-on practical experience inserting the ML 375 using ZOE $\downarrow$ Pelvic Examination Trainer models (ZOE). The trainers then returned to their respective districts and were responsible for training both providers at the $\mathrm{DH}$ and PHC on ML 375 insertions and the community motivators and counselors on IUD counseling and demand generation. Participants for each type of training were selected by service managers at their facilities and all insertion providers were required to have previous CuT $380 \mathrm{~A}$ IUD insertion training.

Insertion providers were trained for one day on: screening clients using the adapted intervention materials; counseling on both the ML 375 and CuT 380A IUDs; counteracting IUD myths and misconceptions; insertion and removal of the ML 375, including infection prevention; managing side effects and complications; conducting follow-up with clients; and management and reporting of client data. The Handbook for Service Providers on IUD were distributed during the training and used as a reference. A total of 127 insertion providers were trained. The motivators and counselors associated with the 12 study facilities and surrounding communities went through a half-day training on introducing IUDs and the ML 375 to community women, counteracting myths and misconceptions, managing side effects, and how to provide referrals for interested women. A total of 427 motivators and counselors were trained. A standard IUD card was used to monitor IUD uptake during the intervention, in the 12 facilities. Up until this point, a standardized system for monitoring and tracking IUD insertions within and across government facilities had not been used. The study's card was developed per current MOHFW recommendations on medical record-keeping and printed in the five languages of the study area.

During the post-intervention phase, we assessed the pilot introduction through in-depth interviews with facility staff $(n=66)$ to learn about their experiences and to identify how the new product could be provided to family planning clients through the existing health system. The post-intervention assessment took place in October 2010 and the data analysis and report writing continued until January 2011.

The post-intervention phase included in-depth interviews with facility staff to assess their perceptions about and experiences with the introduction of the ML 375 . Five staff members at each facility were interviewed in October 2010, for a total of 60 interviews held across the 12 introduction facilities, along with six chief district medical officers in the six districts. We attempted to interview the same respondents during the post- assessment as were interviewed during the preassessment; 36 of the 60 providers were able to be interviewed at both time points. Service statistics collected from the intervention through an IUD card were also reviewed. Similar to pre-intervention phase the tools were developed by FHI360 and pretested in one of the districts in a DH in Uttar Pradesh. The data collection, transcription, translation and data entry were undertaken by a research agency, with FHI conducting analysis.

FHI360 also conducted monitoring visits to the 12 sites from time to time during the intervention to ensure smooth functioning of the intervention. Both quantitative (service statistics) and qualitative data (in-depth interviews) were collected for this assessment and were analyzed using SPSS 18.0 for the service statistics and Atlas/ti 5.0 for the post assessment qualitative data.

The assessment received ethics clearance from FHI 360's Protection of Human Subjects Committee and from the Institutional Review Board (Futures Group International) based in India.

\section{Results}

\section{IUD service statistics}

During the three-month intervention period, IUD providers filled out 597 IUD cards, tracking insertions in the 12 participating facilities. Over this time, the cards showed $479 \mathrm{ML} 375$ s (80\%) and $118 \mathrm{CuT}$ 380 A IUDs (20\%) were inserted. Table 1 shows the distribution of IUD insertions across the six study sites (Table 1). The largest number of ML 375 insertions were done in Kamrup (120), and the fewest in Mysore (29). Different districts appeared to show preferences toward one type of IUD or another (data not shown), with the ML 375 preferred in Gandhinagar (85\% more), Hazaribagh (80\%), and Kamrup (62\%).

Among the two types of facilities where insertions took place, each provided the same percentage of ML 375 versus CuT 380A (DH: 39\%; PHC 61\%). Additionally, auxiliary nurse midwives (ANM) were inserting the most IUDs of both types, although more so for the CuT $380 \mathrm{~A}$.

It should be noted that having more ML 375 inserted during the intervention period should not be taken to represent a preference for one IUD over the other. In Varanasi, providers rarely used the IUD cards to record their CuT 380A insertions, complaining that their reporting burden would be greater as a result of their high CuT $380 \mathrm{~A}$ client load. Additionally, CuT 380A stockouts were noted during the intervention period in several of the facilities; in one facility, the CuT

Table 1. Distribution of all IUDs inserted by district, facility type, and provider [1]

\begin{tabular}{|c|c|c|}
\hline & ML 375 & CuT 380A \\
\hline & $(\mathbf{n}=\mathbf{4 7 9 )} \%$ & 5 \\
\hline District & & 13 \\
\hline Nadia & 19 & 18 \\
\hline Gandhinagar & 19 & 63 \\
\hline Hazaribagh & 18 & 0 \\
\hline Kamrup & 25 & 1 \\
\hline Mysore & 6 & \\
\hline Varanasi & 13 & 39 \\
\hline Facility Type & 39 & 61 \\
\hline DH/FRU & 61 & 60 \\
\hline PHC & & 24 \\
\hline IUD Inserted by & 43 & 10 \\
\hline ANM & 9 & 6 \\
\hline Doctor and medical Officer & & \\
\hline Nurses & & \\
\hline FHW \& LHV & & \\
\hline 1$]$ Data are from IUD cards. & & \\
\hline
\end{tabular}


380A stockouts were acute. Stockouts of the ML 375 were not an issue during the introduction because they were supplied by the intervention agency, independent of the public-sector supply chain, and the program officers monitored supplies.

During the early phase of the intervention, providers reported a lack of clarity on whether the ML 375 insertions should be added to their monthly IUD reporting. This issue was reviewed with state government officials, and the facilities were subsequently granted permission to include the ML 375 insertions in their monthly IUD reporting.

\section{Interviews with providers}

During the post-intervention phase, 60 providers were interviewed, 36 of whom were also interviewed during the pre-intervention phase. Most providers were Auxiliary Nurse Midwives (ANMs) ( $n=15)$, obstetricians and gynecologist (Ob/Gyns) $(\mathrm{n}=13)$, and Medical Officer (MOs) $(\mathrm{n}=11)$.

\section{Provider perceptions on the ML 375}

After three months of offering the ML 375 IUD in their facilities, providers were asked about their perceptions of the new IUD. Most of the providers $(n=43)$ had a positive opinion about the ML 375 as an IUD and family planning method, citing general IUD benefits like an extended effectiveness period (as compared to condoms or oral contraceptives), that it is not permanent, and that few acceptors complain of side effects. From a service provision perspective, providers specifically liked the ML 375 because the IUD comes preloaded on the inserter, resulting in no-touch insertions $(n=19)$. Moreover, providers found the ML 375, as compared to the CuT 380A, easy and less time consuming to insert $(n=37)$ and its soft and different shape reduced the possibility of complications $(\mathrm{n}=32)$.

Providers' comments: I feel it is easier to insert the Multiload. It is very convenient and effective. It is safe. It doesn't cause irritation and is very flexible. $-\mathrm{MO}, \mathrm{DH}$.

At the time of insertion of Multiload 375, we felt it is easy and the material is made of plastic, so it is easy to insert. It is like readymade and so it does not take much time. We like to provide it. -ANM, PHC.

\section{Multiload counseling}

Health care providers were asked to discuss their experiences on counseling clients about ML 375 . Service providers generally found counseling about ML 375 to be a challenge because of widespread myths and misconceptions surrounding IUDs in general, along with low levels of knowledge about the different types of IUDs and the low literacy among potential clients seeking services. Just over half of the service providers $(n=36)$ reported that women were not aware of the ML 375 before coming to the facility, and thus most women were not familiar with how it differed from the CuT 380A, particularly the difference in duration of pregnancy protection.

Providers' comments: Here, the literacy level among patients is lower. There are more myths prevailing regarding IUD so, we have to clear their concerns during counseling. -OB/GYN, DH. They cannot say about Multiload from their own. They only say they are ready for the 5 year Cu T 380A. Actually, we do mention regarding the Multiload first. They don't say anything. -ANM, DH.

Providers in Varanasi reported different experiences in counseling clients. Here, many clients did know about the ML 375 before arriving at the facility, because the ML 375 has been socially marketed for the private sector. Client awareness about the ML 375 was high, and providers reported that clients preferred it over the CuT 380A because they perceived it to be a better IUD.

Providers' comments: People are crazy about Multiload. Maybe because of its effect. They also ask for Multiload and we also tell them about all the options such as Multiload 375 and CuT 380A. In the advertisement they tell good things about Multiload. CuT 380A is also good but people think Multiload is better. $-\mathrm{MO}, \mathrm{DH}$.

When it comes to recommending the ML 375 to clients, few providers considered it suitable for all types of women. Providers identified it as a good method for women with spacing needs, as well as for women who are unwilling to use other family planning methods. Additionally, they believed women with one or two children were candidates for the ML 375 but felt that women with more than two children should be offered sterilization instead. Opinions on eligibility did vary considerably among providers; however, perspectives were generally consistent with opinions expressed overall about the IUD during the pre-intervention assessment.

\section{Multiload insertion process}

When questioned about the specific process of inserting the ML 375 , nearly two-thirds of the service providers $(n=37)$ reported that the insertion process is easier and less time- consuming than the CuT $380 \mathrm{~A}$ process. This was frequently attributed to the ML 375 being preloaded on the inserter inside the package, resulting in no additional time spent on preparing the IUD for insertion. In addition, many providers saw this minimal handling as a benefit for potentially limiting infections. Half of the providers $(n=30)$ noted that clients complained less frequently of pain and discomfort during and after the ML 375 insertions. Four providers did complain about the flexibility of the ML 375 insertion tube, saying that it could cause problems during the insertion process, requiring the providers to discard the IUD and begin the insertion again with a new IUD. Discarded or "wasted" IUDs were tracked throughout the intervention, and there was little recorded wastage, which suggests that while the flexibility of the insertion tube is a concern, it was not a widespread and common problem.

Providers' comments: The process is very good, actually better than $\mathrm{CuT}$ 380A. For $\mathrm{Cu}$ T the loading of the device takes much time and very critical to avoid touch, but for Multiload it is ready. Just open and insert. So, it takes less time than CuT 380A. -MO, PHC.

We can insert it easily. There is no tension about inserting it. Earlier, I used to fear that it may be misplaced but, after the training, I have confidence. Complaints are very less, slight lower abdomen pain. FHW, DH.

\section{Follow-up of IUD insertions}

The MOHFW policy on following up IUD insertions recommends that a client return to the facility for a follow-up exam after her first monthly bleeding or four to six weeks after the IUD insertion. Subsequent visits should be made once a year. This follow-up policy was part of the ML 375 IUD introduction and was included in the Handbook for Service Providers on IUD. Qualitative data on IUD follow-up, whether ML 375 or CuT 380A, was extremely limited; however, information collected during the study suggests providers lack a clear and well-defined strategy for routinely following up IUD insertions, despite the MOHFW policy.

Providers' comments: "But there are some women they did not come yet. They will also come as per their requirement or convenience. The reason behind this is that if they are feeling no problem then they 
will not come for check-up as they come from interiors and it involves cost. -ANM, PHC.

The IUD card did track how many IUD acceptors returned to the facility where they had their IUD inserted; however, those who returned to alternate facilities or who were visited in their home or other community location were not tracked by the card. Overall, follow- up visits were only recorded among ML 375 acceptors, and just $8 \%$ of these acceptors $(n=31)$ returned to the facility for a first visit; there were no repeat visits. These few women returned on average one month $(32.2$ days) after their ML 375 was inserted, although visits ranged from 7 to 71 days afterward. Of the 31 ML 375 users who made a follow-up visit, most complained of bleeding (61\%) and pain (42\%). Only one woman of the 597 tracked by the IUD card was documented to have her IUD, an ML 375, removed during the intervention period.

\section{375 introduction training}

Of the 60 providers interviewed, 47 (78\%) had participated in one of the ML 375 trainings; participation was not an eligibility requirement for the interviews. The majority of the 47 had attended the Training of Providers for Multiload Insertion $(n=41)$, whereas the remaining attended the Training of Motivators and Counselors $(n=4)$ or were ML 375 Master Trainers $(\mathrm{n}=2)$.

Providers and motivators who were part of the Multiload introduction trainings were asked about their experiences during the training and about what new information they learned. Participants reported that the details of the ML 375-including shape, duration of effectiveness, and side effects-were new to them. Insertion providers also discussed what they learned about the insertion process, such as the fact that loading the ML 375 on the inserter is not required and that an adaptor is unnecessary.

After the training, most of the participating providers and motivators felt confident about counseling clients on the ML 375; and the insertion providers were comfortable with inserting and removing the IUD. When asked about suggestions for improving the training, the providers reported that the training duration should be increased to include supervised practice insertions on patients, as opposed to only on ZOE models $(\mathrm{n}=17)$.

Moreover, motivators and providers recommended incorporating audio-visual materials into the trainings.

Providers' comments: The new information in the training was related to IUD counseling. Specific counseling has to be given... we have to tell them about the side effects, the benefits of the product which we are inserting. $-\mathrm{OB} / \mathrm{GYN}, \mathrm{DH}$.

The training was very compact, and I feel it could have been extended to two days for better perception. One day reserved for counseling and one day for the insertion part. Real life practice can be done for counseling and it is very difficult for real life insertion practice. Models can be provided for practice session for all participants. - MO, PHC.

\section{Intervention materials}

During their interviews, all providers were asked if they had received and used the training and communication materials developed for the ML 375 introduction. About two-thirds had received all of them and slightly fewer used them in the three months of the intervention (Figure 1).

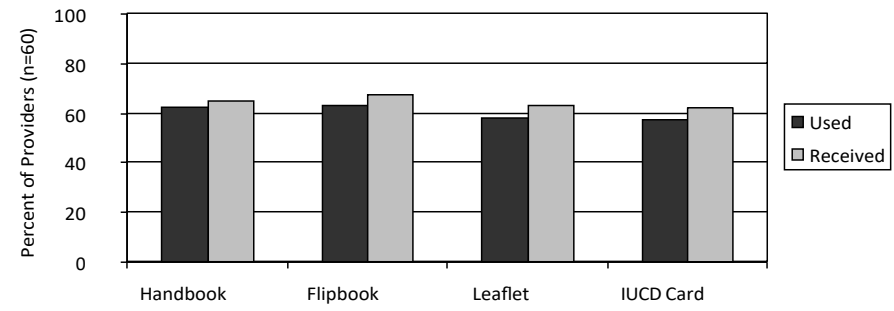

Figure 1. Intervention materials received and used by providers

Of the four materials introduced, the IUD card was used on a daily or near-daily basis by the providers inserting IUDs, both ML 375 and $\mathrm{CuT}$ 380A. Overall, providers found the card a helpful record of what type of IUD a woman had inserted and when the insertion occurred. During monitoring visits, some providers did raise concerns about the additional reporting burden these cards placed on them. In Varanasi, where high client loads were seen for CuT 380A IUDs, the reporting burden was so great that providers only used the cards to track ML 375 insertions; they felt that tracking CuT 380A insertions was too timeconsuming.

Providers also said that they thought the perforated counterfoil that could be given to the clients was useful. Providers would have liked a space to record the client's name on the IUD card, which was not included during the study for ethical reasons, to facilitate client tracking and follow-up both within the facility and in the community by accredited social health activists (ASHA, community-based workers).

Providers' comments: The card is prepared systematically, the follow up date is mentioned, so we can emphasize them to come for follow up, and the five warning signs have been demonstrated by pictures, so the patient can make out that she needs to take advise in such condition. So, it is helpful. -OB/GYN, DH.

It is quite good; all the required information could be recorded at the card as per the instruction given. It is good that we can give a record to the client also. It is also readymade, and we need not to maintain a different copy as we used to do it for CuT 380A earlier. -ANM, DH.

\section{Barriers for ML 375 access, uptake, and use}

Health care providers were questioned about what they perceive to be major barriers limiting the access, uptake, and use of the ML 375. Within the facilities, providers identified barriers that were not solely confined to ML 375 provision, but provision of all IUDs in the facility. Issues like power failures, poor infrastructure, and lack of necessary instruments were common. For example, in the Kamrup, Nadia, and Mysore facilities, providers cited an acute lack of space and limited sets of instruments that hampered the number of insertions that could be undertaken, leading to long waiting times. Providers' inability to handle high client load for family planning services also affected the acceptance of IUDs, including ML 375.

Providers' comments: Only if we have our own set of equipments for insertion and all then we need not to depend on the OT instruments and the waiting time of the patient's decreases and the process become faster. - MO, DH.

Providers reported that myths and misconceptions, along with low awareness of IUDs in general, limited client uptake of the ML 375. According to the providers, women's decisions about adopting the ML 375 are guided by their overall preference for or aversion to the IUD in general, as few are aware that there are multiple IUD options. 
With the CuT 380A already available in the public sector, many women's exposure to the IUD is through hearing about it and its side effects, whether truth or myth. Providers reported that clients often assume that all types of IUDs have similar side effects and efficacy profiles, which posed a challenge to providers who tried to differentiate the ML 375 and discuss its benefits, let alone dispel myths like that the IUD will move around in the body or cause infertility. Additionally, providers were faced with overcoming the misconceptions of clients' husbands and mothers-in-law, who often must approve of a woman's use of family planning and can influence her method selection.

Providers' own misconceptions, insufficient knowledge, and lack of counseling skill on informed choice are also barriers to uptake of ML 375 and IUDs overall. While providers were not asked to comment on their own performance, it became apparent through the interviews that some providers lacked these necessary skills. Both before and after the intervention, providers could not fully cite accurate IUD eligibility criteria and exhibited bias in their consideration of who was suitable to receive an IUD. When providers were asked to discuss their experiences on counseling clients about the ML 375, discussions on side effects were limited or incorrect, thus not fully preparing clients on what to expect, thereby increasing the likelihood of discontinuation.

\section{Facility- and community-based services required}

The most frequently requested change in the ML 375 intervention was the provision of incentives to motivate clients to use the ML 375. In some areas, incentives were available in the private sector for the ML 375. Providers repeatedly emphasized that these IUD incentives should be consistent across both the private and public sectors to prevent competition for IUD services.

Providers in some facilities reported the need separate rooms for IUD insertion, aside from operating theatres, postpartum units, and labor rooms, where IUD insertions have less priority. Additionally, they suggested that more private space be allocated for family planning counseling. The lack of space and privacy created significant concerns during the intervention since they led to longer waiting time for clients and indicated an inability to handle larger volumes of clients if the program were to be rolled out as a routine service. Separate insertion and counseling space could alleviate this burden. Some providers requested a more formal supervisory system to monitor and ensure service delivery.

In several of the districts, providers discussed the benefits of having ASHA and other community-level staff actively promoting the ML 375 in the surrounding communities. These motivators were introducing the ML 375 to women in their homes and during village health days and encouraging interested women to visit the facilities for more information and IUD uptake. While it was often reported that the clients could not specifically name the ML 375, women could specify their interest in the "5-year IUD".

Providers' comments: Yes, because our ANMs and fieldworkers counsel them on their home visit and motivate them before sending to this facility. They know that the new copper $\mathrm{T}$ for 5 years and the older one is for 10 years. They cannot take the name of Multiload or mention any number also. They know it from the ANMs that its safe. -Nurse, DH.

\section{Discussion}

The post-intervention assessment highlights various operational issues for the MOHFW to consider in scaling up the introduction of the ML 375 into additional public sector health facilities. The operational issues examined through this qualitative assessment include various ML 375 service delivery aspects such as counseling, service provision, following up of clients, training, intervention communication materials, barriers, and necessary facility- and community-based services. The overall feedback from providers, both those who inserted ML 375s and the community motivators, was positive and encouraging about further introduction. However, the assessment also exposed weaknesses not only in the ML 375 introduction, but in overall IUD provision in the 12 facilities. There is a need for a structured referral system to manage serious complication or offer higher levels of care for all IUD clients at PHCs.

Provider feedback, service statistics from the IUD card, and observations from study monitoring visits found that after one day of ML 375 training, providers with existing experience in inserting CuT 380A IUDs are able to successfully counsel on and insert ML 375s with limited reports of clinical complications or other problems. Providers did recommend a longer training, particularly the ANM, which would include supervised ML 375 insertion on clients. A longer training could also accommodate more time for dispelling providers' misconceptions of all IUDs, particularly eligibility criteria, and improving balanced and informed counseling on family planning options. Aside from some providers' misconceptions and insufficient counseling skills, most of the barriers hampering ML 375 provision and uptake had less to do with the providers and are more a result of facility- and community-level challenges that are relevant to all IUD provision. Facility constraints like sharing space with services that take greater priority, limited privacy, insufficient equipment, and likely supply chain issues all limit women from receiving IUDs. CuT 380A stockouts were noted during the intervention period in several of the facilities.

If the ML 375 IUD is to be considered a viable option for limiting and spacing births, the MOHFW, both at national, state, and district levels need to facilitate all IUD provision. If clients can receive incentives for adopting the ML 375 in the private sector, women lack motivation to choose the ML 375 from the public sector. (Note: Cash and kind at an incentive is available in the private sector in India for IUCD. However, in public sector cash incentive is available for accepting sterilization but not for IUCD).

Staff turnover at the 12 facilities and surrounding communities was a problem over the course of the intervention period. Staff who underwent ML 375 orientation and training were transferred or discontinued service in two sites, leaving gaps in numbers of staff qualified in ML 375 provision.

In spite of these challenges, the ML 375 was well received in the 12 facilities and their surrounding communities. With improvements in the intervention and appropriate health system infrastructure and policy modifications, the ML 375 has the potential to be an important addition to the family planning method mix available under the FWP.

The study had many limitations. The aim of this study was to introduce the ML 375 IUD into 12 public sector facilities already offering the CuT 380A IUD, and to assess the operational challenges related to its introduction. The post-intervention assessment was only qualitative in nature and only used provider feedback to understand the operational challenges.

The primary limitations are a result of the study design. First, feedback from the women accepting the ML 375 was not collected, preventing the study from assessing the clients' perspective on the 
ML 375 introduction, including the counseling she received and her experience using this IUD type. Second, the study was not designed to evaluate the impact of the ML 375 introduction on overall IUD provision in the 12 facilities.

Therefore, no conclusion can be made whether the availability of the ML 375 increased IUD uptake or just gave women who would have otherwise chosen the CuT 380A another IUD option. Third, facility selection was purposive and only included $\mathrm{DH}$ and $\mathrm{PHC}$ facilities and, in some cases, facilities with previously high IUD client loads. The application of these results to other facilities should be done with these limitations in mind.

Additionally, this intervention did not focus greatly on demand generation for the ML 375, with mass media activities or community promotion beyond the ML 375 leaflets for users and work of the ASHA and ANM. Stock out of $\mathrm{Cu}$ T 380A during the intervention period, health facility staff's disinterest to fill up the IUD cards and providers lacking clarity on whether the ML 375 insertions should be added to their monthly IUD reporting posed a challenge during the pilot introduction.

\section{Funding support}

This study was made possible by the generous support of the American people through the United States Agency for International Development (USAID) under the terms of FHI Cooperative Agreement No. GPO-A-00-08-00001-00, the Program Research for Strengthening Services (PROGRESS) project. The opinions expressed herein are those of the authors and do not necessarily reflect the views of USAID.

\section{Acknowledgement}

We especially thank Dr. Loveleen Johri, senior reproductive health advisor, PHN from USAID/India, for her technical insights and support throughout the assessment period. We acknowledge Dr. Irina Yacobson, assistant medical director, FHI 360/NC for technical review of the intervention materials. Eva Canoutas, associate director,
FHI 360/NC, supported research utilization activities and contributed to the preparation of the final report. We express gratitude to the State Government's Family Planning department of Assam, West Bengal, Jharkhand, Uttar Pradesh, Gujarat, and Karnataka, which allowed us to implement the intervention. We are grateful to the Government of India, especially Dr. Kiran Amwani, Dy. commissioner (FP), Ministry of Health and Family Welfare, Family Planning Division, and Dr. Jaya Lalmohan, team leader and national consultant, Family Planning Division, for their continuous support throughout the implementation period.

\section{References}

1. Ministry of Health and Family Welfare Government of India. Repositioning IUD in The Family Welfare Programme: Strategy, Operational Plan and Achievements. In: Division FP, editor. New Delhi 2008.

2. International Institute for Population Sciences (IIPS) and Macro International. Nationa Family Health Survey, 2005-2006: India. Mumbai, India: International Institute for Population Sciences 2007.

3. Pariani S, Heer DM, Van Arsdol MD Jr (1991) Does choice make a difference to contraceptive use? Evidence from east Java. Stud Fam Plann 22: 384-390. [Crossref]

4. Ross J, Hardee K, Mumford E, Eid S (2002) Contraceptive method choice in developing countries. Int Fam Plan Perspect 28:32-40.

5. Seiber EE, Bertrand JT, Sullivan TM (2007) Changes in contraceptive method mix in developing countries. Int Fam Plan Perspect 33: 117-123. [Crossref]

6. d'Arcangues C1 (2007) Worldwide use of intrauterine devices for contraception. Contraception 75: S2-7. [Crossref]

7. Salem R (2006) New attention to the IUD: expanding women's contraceptive options to meet their needs. Population reports Series B: Intrauterine devices:1.

8. Khan M, Patel B, Gupta R (1999) The quality of family planning services in Uttar Pradesh from the perspective of service providers. In Koenig A Michael and Khan ME: Improving Quality of Care in India's Family Welfare Programme. New Delhi: Population Council, 1999. p. 238-69.

9. Santhya K. Contraceptive use dynamics. In: Jejeebhoy S, editor. Looking back, looking forward: a profile of sexual and reproductive health in India: Rawat 2004.

10. Iyengar K1, Iyengar SD (2000) The Copper-T 380A IUD: a ten-year alternative to female sterilisation in India. Reprod Health Matters 8: 125-133. [Crossref]

Copyright: (C)2018 Basu S. This is an open-access article distributed under the terms of the Creative Commons Attribution License, which permits unrestricted use, distribution, and reproduction in any medium, provided the original author and source are credited. 